Mariângela Gagliardi Caro Salve

Antonia Dalla Pria Bankoff'

\section{Análise da Intervenção de um Programa de Atividade Física nos Hábitos de Lazer}

'Faculdade de Educação Física da Universidade de Campinas, Brasil
O presente trabalho teve por objetivo analisar a intervenção de um programa de atividade física nos hábitos de lazer, ou seja, investigar quais as atividades físicas, preferidas, executadas por trabalhadores do setor da marcenaria da UNICAMP, nas horas do lazer. Selecionamos 40 voluntários ( $n=40$ ), do sexo masculino. Elaboramos e aplicamos um programa de atividade física praticada durante o expediente de trabalho, e abordamos questões de como ter hábitos de vida saudáveis, incluindo recomendações sobre a importância prática da atividade física nas horas de lazer. Este teve a duração de 8 meses, com sessões de uma hora, duas vezes por semana. Chamamos de grupo II $(n=10)$ aos que acompanharam o programa até o quinto mês, mas mantivemos contato com esse grupo, orientado-os nas questões acima abordadas até o final do programa. Denominamos grupo III $(n=30)$ os que freqüentaram até terminar o programa.

Palavras-chaves Atividade Física, Lazer, Saúde, Qualidade de Vida.

The purpose of this work was to assess how a physical activities program affects recreational habits, in other words, to determine the nature of the preferred recreational physical activities of carpentry workers at UNICAMP (UNICAMP - University of Campinas, State of São Paulo, Brazil).Accordingly, we selected 40 male volunteers $(n=40)$ and we elaborated a physical activities program that was implemented during working hours. We also dealt with issues relating to healthy living practices, including recommendations concerning the importance of practicing physical activities during recreation time. This program lasted 8 months, with two one-hour sessions each week. A group of 10 workers, denominated Group II, participated only to the fifth month, however we maintained contact with its members and oriented them regarding the aforementioned issues throughout the full program term, we called the volunteers that completed the program Group III ( $n=30)$.

Keywords Physical Activity, Leisure, Health, Quality of Life. 


\section{Introduçao}

Conforme vários estudos mostram a prática regular e sistematizada da atividade física é fundamental na prevenção e controle das doenças crônico-degenerativas. Porém, ainda é grande a porcentagem de população inativa, ou seja, sedentária.

Para CARVALHO (1995) embora a maior parte da população adulta possa ser classificada como inativa, somente 15 a $25 \%$ seria considerada fisicamente ativa de forma regular. O sedentarismo é considerado como uns dos principais causadores de doenças crônico-degenerativas, como a hipertensão, obesidade, diabetes, lombalgias, entre outras. Existem claras evidências de que o sedentarismo seja o principal fator de risco coronariano, já que é relativamente alto e sua prevalência é bastante superior a dos outros fatores (idade, sexo, história familiar...). Com a inatividade física o corpo responderá atrofiando a massa muscular, perdendo a resistência, diminuindo a massa óssea, as funções pulmonares, a flexibilidade e outras capacidades físicas.

Dados epistemológicos mostram que a inatividade física é, provavelmente, um fator de risco individual para causar a hiperlipidemia, a hipertensão, a obesidade e o diabetes. As pessoas inativas, em geral, são mais hipertensas, hiperlipidêmicas, com maior porcentagem de gorduras e propensão de tornarem-se diabéticas não dependentes de insulina. Ao passo que, as pessoas com um estilo de vida mais ativo, têm maiores possibilidades de prevenir essas patologias, ACHOUR (1996).

RAMOS (1998) verificou, através de questionário composto de 14 questões, entre 246 alunos da UNICAMP, sendo137 homens e 109 mulheres, que apenas $28,86 \%$ dos alunos que entram na universidade apresentam vida ativa, fisicamente, ou seja, realizam exercícios, três ou mais vezes por semana, e 32,93\% são considerados intermediários, isto é, praticam atividade física uma ou duas vezes por semana.

Segundo BARBANTI (1991) 67\% da população adulta brasileira não realiza nenhum tipo de atividade física. MATSUDO et al. (1996) mencionam que pesquisas recentes mostram, que nos Estados Unidos e na Inglaterra, a taxa de inatividade física é de $24 \%$ e $15 \%$ respectivamente. Epidemologistas americanos, segundo estes autores, estimam que há, aproximadamente, 250.000 mortes por ano que poderiam ser atribuídas à inatividade física e 32.400 vidas poderiam ser salvas se houvesse incremento na atividade física. O Projeto Saúde (um estudo realizado pelo $\mathrm{Mi}$ nistério da Saúde sob estilo de vida) revelou que $96 \%$ das pessoas não têm um estilo de vida inteiramente saudável, somente $10 \%$ da população, entre 18 e 55 anos, declaram fazer atividade física regularmente, pelo menos, três vezes por semana. Estudos realizados por MATSUDO et al. (1996), no CELAFISC, em escolares de São Caetano do Sul e llha Bela revelaram que os hábitos de atividades físicas das crianças brasileiras são menores e gastam mais tempo assistindo TV que as crianças americanas.

Atualmente, nota-se o aumento do número de adolescentes sedentários e mais obesos (MOUSSA et al. 1994). O hábito de assistir televisão tem sido o mais comum entre os adolescentes, existe uma relação positiva entre o nível de adiposidade e o número de horas diárias de TV assistidas (MATSUDO, et al. 1997). O problema agrava-se mais quando há o consumo de alimentos, enquanto se assiste TV (BAR-OR, et al.1998).

De acordo com LITVAK et al., (1987) os hábitos sociais, fumo, ingestão de álcool e de cafeína, associados ao estresse e à inatividade física, contribuem para a instalação de cardiovasculares, doenças pulmonares obstrutivas crônicas, neoplasias, diabetes mellitus e cirrose. Essas doenças vêm ocupando um maior espaço de morbimortalidade nas populações latinoamericanas.

De acordo com RIGATTO (1994), antigamente, a atividade física era considerada uma maneira de gastar o tempo mais depressa. Hoje, já se comprovou que, se realizarmos exercícios físicos, teremos até quatro vezes mais energia vital no corpo, do que se tivermos uma vida sedentária.

Nas sociedades industrializadas, as atividades físicas têm representado, cada vez mais um fator de qualidade de vida, tanto de forma geral, como em relação à saúde 
das pessoas, em todas as idades e condições, estando associada à maior capacidade de realização de trabalho físico e mental, com mais entusiasmo, sensação de bem-estar, menor gasto com saúde e diminuição do risco de doenças crônico-degenerativas (BLAIR, 1993).

São vários os estudos que indicam benefícios, tanto nos aspectos psicológicos como biológicos da atividade física, ou seja, melhoria da capacidade cardio-respiratória, diminuição de sintomas vasomotores, da pressão arterial, da incidência de doenças cardiovasculares, retardamento e prevenção do aparecimento de diabetes melittus, da osteoporose, da obesidade, da hipertensão, redução da ocorrência de certos tipos de câncer, aumento da expectativa de vida, entre outros. No aspecto psicológico ocorre melhoria da auto-estima, autoconceito, auto-imagem e diminuição da ansiedade e depressão (CORBIN \& LINDSEY 1997; DISHMAN, 1994; NIEMAN, 1998; BLUENTHAL et al., 1988; CREWS \& LANDERS, 1987; SINYOR, et al. 1983; GUTHRIE, et al.1995).

Para RIGATTO (1994) o exercício físico é o melhor tranquiilizante que o homem encontra na natureza, pois a morfina endógena, produzida pelo cérebro na atividade física, é colocada no sangue e circula por todo o corpo cerca de 24 horas após o exercício. Para Carvalho (1995), o exercício físico é um tranqüilizante natural.

O exercício físico diminui os sintomas de estresse, ansiedade, depressão e raiva, bem como a influência de estressores psicossociais no indivíduo (BLUMENTHAL et al., 1998; CREWS \& LANDERS, 1987 e SINYOR et al., 1993).

STEPTOE (1994) comprovou que quanto melhor foi o condicionamento físico dos indivíduos tanto mais favoráveis serão as suas respostas ao estresse.

GUISELINI (1996) afirma que o corpo humano é uma máquina de fazer inveja ao mais sofisticado computador. É constuido por 220 ossos, 400 músculos, $1000 \mathrm{Km}$ de artérias, sendo impulsionado pelo músculo cardíaco que pulsa cerca de 60 vezes por minuto, 3600 vezes por hora. Esse conjunto requer cuidados, dedicação e procedimentos.

O autor sugere os seguintes procedimen- tos necessários para a sua perfeita manutenção:

- decisão as pessoas devem convercer-se de que cuidar do corpo é algo prioritário;

- prazer devem-se convercer que cuidar do corpo é algo agradável;

- escolha de atividade - é necessário procurar uma atividade que não apenas fortaleça os músculos, diminua a porcentagem de gordura, mas também beneficie o lado emocional;

- respeito ao próprio corpo- é necessário não se tornar um verdadeiro escravo do treinamento.

Existem várias opções de atividades físicas que promovem bem-estar físico e mental no organismo humano.

KING et al. (1993) revelaram aspectos positivos do exercício aeróbico na redução dos níveis de ansiedade, de depressão e de estresse.

SAMULSKI \& LUSTOSA (1996) relataram a influência do exercício aeróbico sobre o stresse, o humor e o autoconceito.

FIATARONE et al. (1990) sugeriram os exercícios de força, flexibilidade, equilíbrio, coordenação e atividades aeróbicas para a melhoria da saúde e bem estar psicológico de adultos.

Para GANDEE (1998) as atividades aeróbicas como caminhada, natação, entre outras, favorecem para tornar o corpo mais relaxado.

MAZARINI (1992) recomendou a prática das atividades aquáticas, pois esta proporciona prazer, bem-estar e os indivíduos descobrem novas maneiras de realizar exercícios físicos e a ficarem mais desinibidos. Os exercícios, no meio líquido, oferecem a oportunidade de as pessoas executarem, livremente, os mais diversos movimentos, graças a flutuabilidade do corpo. Essa movimentação é beneficiada quando a temperatura mantiver-se em torno de 28 a 34 graus centígrados. A água aquecida proporciona aumento da circulação sanguínea e da sensibilidade neuro-sensorial, associada à redução dos efeitos da ação da gravidade, que são responsáveis pela melhora do tônus muscular postural. Nesse caso, favorecem uma adequada mobilidade articular e a elasticidade muscular.

CAMPOS \& POPOV (1998) mencionaram que com a prática da hidroginástica as doenças, como a osteoporose, são altamente 
controladas, tanto em termos de prevenção, como de tratamento. Outra atividade importante é a caminhada, pois essa contribui para melhorar a atividade do sistema nervoso, cardiovascular e respiratório do organismo. É indicada para pessoas que estão iniciando um programa de atividade física, indivíduos obesos, idosos e para os que possuem problemas no sistema locomotor.

RIPPE et al (1998) relataram que a prática da atividade física moderada, como a caminhada, provoca redução do aparecimento de doenças do coração, contribui para a diminuição da ansiedade, tensão, controle do índice do colesterol, hipertensão e diminuição na velocidade da instalação da osteoporoe.

Os exercícios respiratórios melhoram e ativam a função da respiração pulmonar e contribuem diminuindo os gastos energéticos do organismo. Os exercícios podem ter caracter estático ou dinâmico, isto é, com presença ou falta de movimentos de braços e tronco, respectivamente. Para executálos é preciso recomendar a respiração nasal, a fim de evitar hiperventilação e diminuição do tempo da respiração (CAMPOS \& POPOV, 1998).

Assim, a atividade física é um dos elementos fundamentais para aquisição e manutenção de uma boa qualidade de vida. A sua prática, nas horas de lazer, é importante no estabelecimento do equilíbrio físico e mental.

Podemos considerar que em décadas mais recentes, segundo NAHAS (1997), a qualidade de vida tem emergido como um fator de destaque em investigações na área da saúde, nos estudos ligados às condições de trabalho e na perspectiva de lazer do ser humano. A atividade física, no lazer, representa um fator importante de qualidade de vida

Para SABA (1998) o conceito de aptidão física, que atualmente se procura adotar envolve todas as dimensões do comportamento humano. Hoje, a aptidão física conversa com outras áreas, dialoga que outros fatores que incidem sobre o homem e a sua natureza. Essa atitude difere daquela anteriormente predominada, que ficava preocupada, somente, em conseguir alguns minutos, a menos, em determinadas práticas. O conceito da aptidão física estava forte- mente associado às variáveis que afetam a performance esportiva, restrita somente a um grupo de pessoas que se dedicavam a essa prática (alguns por profissão, outros poucos, por lazer). A consciência da aptidão física que se tem hoje é que há outros elementos que ultrapassam o potencial hereditário, elementos que dependem da oportunização da prática de qualquer atividade física para o desenvolvimento das capacidades físicas. Assim, hoje, aptidão física está relacionada aos aspectos que levam à melhoria da qualidade de vida. A busca por uma saúde melhor, por uma vida saudável, eis o desafio que percorrem pessoas que abandonam a vida sedentária, em busca de um outro universo referencial. É necessário que se criem condições e espaços adequados para se alcançar esses objetivos.

AMERICAN HEART ASSOCIATION, SUBCOMMITTEE ON EXERCISE/CARDIAC REHABILITION (1981) recomenda a pratica da atividade física, independente da idade, do sexo e do local, com segurança e orientação profissional, de forma regular e sistematizada e por toda a família. É importante constar com mudanças nos hábitos alimentares, reeducação no que diz respeito às atividades realizadas no dia-adia, como jogar vídeo-game, navegar pela internet muitas horas seguidas, utilização de elevadores no lugar de escadas, etc. A saúde deve ser considerada de forma mais abrangente, envolvendo bem-estar físico, psíquico e social. A prevenção ou tratamento das doenças crônico-degenerativas vai se estabelecer a partir da identificação e remoção dos agentes causadores por essas doenças, chamados de "fatores de riscos". O treinamento físico protege o desenvolvimento das doenças coronarianas, além de garantir a sobrevida após um ataque cardíaco.

Nesse sentido, a consciência dos benefícios da prática regular ajuda em programas de atividade física (SAMULSKI \& LUSTOSA, 1996).

O NIH CONSENSUS CONFERENCE (1996) propõe que sejam desenvolvidas estratégias para encorajar indivíduos, com diferentes experiências e conhecimentos, a admitir e aderir a um estilo de vida ativo.

DISHMAN (1993) considera que mesmo em países desenvolvidos, através de infor- 
mações eficientes para conscientizar a população dos malefícios causados pela vida sedentária, grande parte da população se mostra insensível.

A consciência do valor da prática da atividade física, seja nas horas de lazer ou no trabalho, é fundamental, tanto em termos de saúde como em qualidade de vida. A seguir veremos o que os autores entendem por lazer.

Segundo DUMAZADIER (1973) lazer é:

"...conjunto de ocupações às quais o indivíduo pode entregar-se, de livre vontade, seja para divertir-se, recrear-se e entreter-se, ou ainda, para desenvolver sua formação desinteressada, sua participação social voluntária, ou sua livre capacidade criadora, após livrar-se ou desembaraçar-se das obrigações profissionais, familiares e sociais".

Para REQUIXA (1980) lazer é "...ocupação não obrigatória, de livre escolha do indivíduo da qual vive e cujos valores propiciam condições de recuperação psicossomática e de desenvolvimento pessoal e social".

MEDEIROS (1980) entende como lazer: "espaço de tempo não compreendido, do qual podemos dispor livremente, porque já cumprimos nossas obrigações de trabalho e de vida".

No conceito de MARCELINO (1987) lazer é uma escolha individual praticada no tempo disponível e de que ocorrem determinados efeitos, como descanso físico e mental, divertimento, desenvolvimento da personalidade e da sociabilidade. O lazer também pode ser considerado como "atitude", assim não abrange somente as situações de prática, uma vez que a atitude independe da situação da prática ou de consumo, ou seja, um indivíduo pode ativamente participar de espetáculo de teatro, fazendo parte ativamente da montagem da platéia.

Diante da necessidade de aquisição de hábitos saudáveis recorremos às recomendações de AMORIM et al. (1997), ou seja, que ao oferecer atividades essas venham de encontro às necessidades e motivações individuais, torna-se fundamental conhecer as experiências de grupos específicos e os seus hábitos, de forma a possibilitar a elaboração de programas de atividades físicas, capazes de estimular a incorporação e manutenção de vida saudável, visando proporcionar um impacto na qualidade de vida.

Esse trabalho teve por objetivo analisar a intervenção de um programa de atividade física nos hábitos de lazer, ou seja, investigar quais as atividades físicas preferidas, executadas por trabalhadores do setor da marcenaria da UNICAMP, nas horas do lazer.

\section{Metodologia}

Elaboramos um programa de atividade física composta por exercícios de alongamentos musculares, relaxamentos, equilíbrio corporal, respiratórios, caminhada e hidroginástica e abordamos questões dos vários hábitos de vida que a tornam saudável, incluindo a importância da prática da atividade física nas horas de lazer. 0 programa teve a duração de 8 meses, com sessões de uma hora, duas vezes por semana.

Também, elaboramos um protocolo de avaliação, composto por vinte e uma questões abertas e fechadas, que foi aplicado antes e após o término do programa.

O programa foi aplicado no Laboratório de Eletromigrafia e Postura, no bosquinho, na piscina da Faculdade de Educação Física e nas dependências da marcenaria da UNICAMP.

Selecionamos 40 voluntários $(G I, n=40)$, do sexo masculino, trabalhadores do setor da marcenaria da UNICAMP, com idade compreendida entre 33 e 60 anos. Chamamos de grupo II ( $\mathrm{Gll}, \mathrm{n}=10)$ aos que acompanharam o programa até o quinto mês, mas mantivemos cantato com esse grupo, orientando-os nas questões acima abordadas até o final do programa e grupo III ( $G I I I, n=30$ ) aos que freqüentaram até terminar o programa. 


\section{Resultados}

Tabela 01 Atividades físicas, preferidas e executadas pelos trabalhadores do setor da marcenaria da UNICAMP, nas horas do lazer, antes e após o programa de atividade física.

Atividades físicas, preferidas e executadas pelos trabalhadores dos grupo I, II e III do setor da marcenaria da UNICAMP, nas horas do lazer, antes e após o programa de atividade física.

\begin{tabular}{|c|c|c|c|c|c|c|c|c|c|c|c|c|}
\hline & \multicolumn{2}{|c|}{ Gl Antes } & \multicolumn{2}{|c|}{ Gl Após } & \multicolumn{2}{|c|}{ Gll Antes } & \multicolumn{2}{|c|}{ Gll Após } & \multicolumn{2}{|c|}{ Glll Antes } & \multicolumn{2}{|c|}{ Glll Após } \\
\hline $\begin{array}{c}\text { Modalida } \\
\text { des }\end{array}$ & F.A. & F.R. & F.A. & F.R. & F.A. & F.R. & F.A. & F.R. & F.A. & F.R. & F.A. & F.R. \\
\hline Futebol & 3 & 7,5 & 17 & 42,5 & 3 & 30 & 2 & 20 & $\cdot$ & $=$ & 15 & 50 \\
\hline $\begin{array}{c}\text { Caminha } \\
d a\end{array}$ & - & - & 30 & 7,5 & - & $=$ & 1 & 10 & - & - & 2 & 6,6 \\
\hline Ciclismo & - & - & 5 & 12,5 & - & - & 1 & 10 & - & - & 3 & 10 \\
\hline Corrida & - & - & 2 & 5 & - & - & - & - & - & $=$ & 9 & 20 \\
\hline $\begin{array}{l}\text { Alongam } \\
\text { ento }\end{array}$ & - & - & 4 & 10 & - & - & 1 & 10 & - & $=$ & 3 & 10 \\
\hline Natação & - & - & 1 & 2,5 & - & - & - & - & - & - & 1 & 3,3 \\
\hline
\end{tabular}

F.A. - freqüência absoluta

F.R.- Freqüência relativa

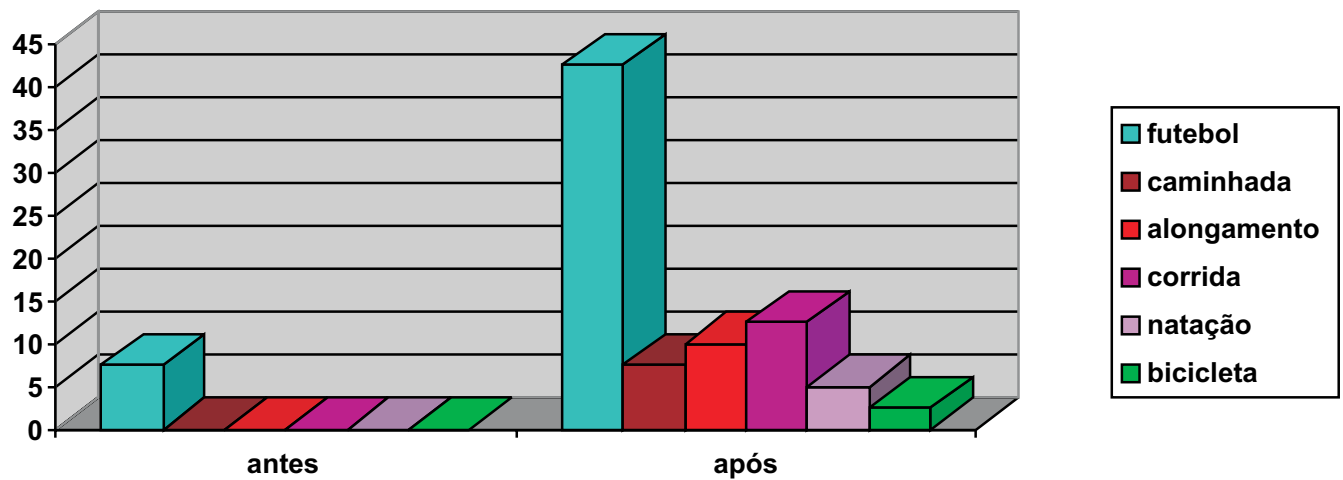

Gráfico 01 Atividades físicas, preferidas e executadas pelos trabalhadores do grupo I do setor da marcenaria da UNICAMP, nas horas do lazer, antes e após o programa de atividade física.

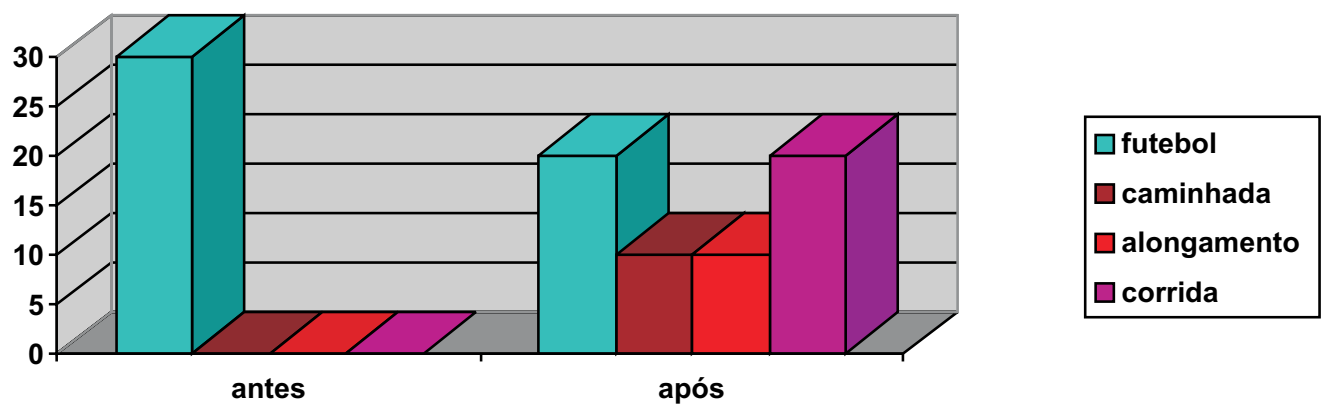

Gráfico 02 Atividades físicas, preferidas e executadas pelos trabalhadores do grupo Il do setor da marcenaria da UNICAMP, nas horas do lazer, antes e após o programa de atividade física. 


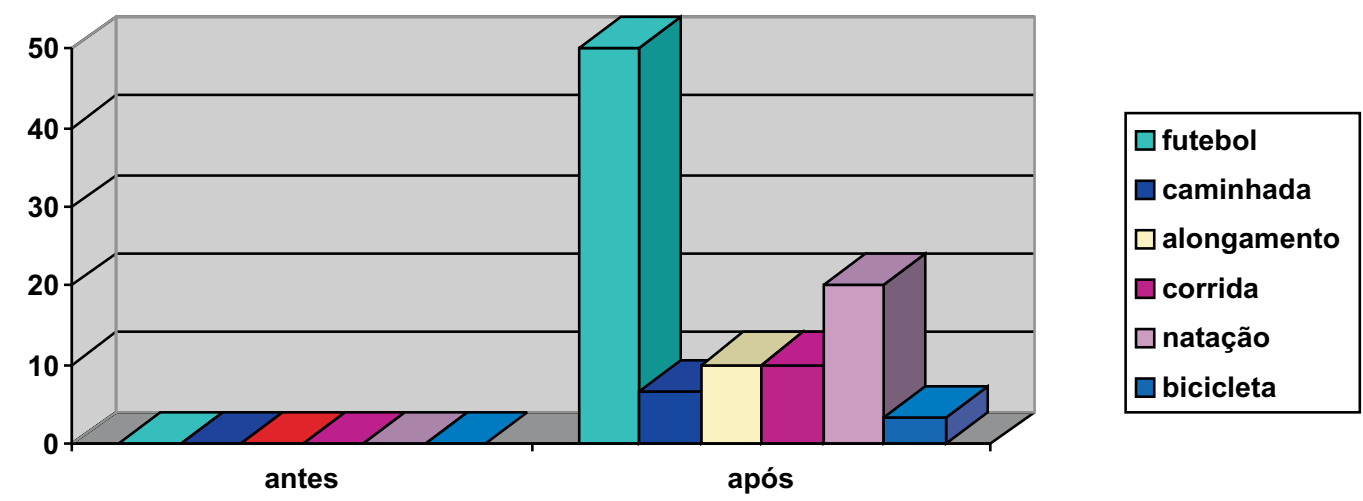

Gráfico 03 Atividades físicas, preferidas e executadas pelos trabalhadores do grupo III do setor da marcenaria da UNICAMP, nas horas do lazer, antes e após o programa de atividade física.

\section{Análise dos resultados}

Observamos que aumentou $o$ interesse e a prática da atividade física nos três grupos analisados.

O grupo I inicialmente somente jogava futebol $(7.5 \%)$, passou a praticar ciclismo $(12.5 \%)$, corrida $(5 \%)$, natação $(2.5 \%)$, fazer alongamentos musculares (10\%), caminhada $(7.5 \%)$ e aumento da porcentagem de praticantes do futebol (42.5\%). O mesmo ocorreu com o grupo II, que só jogava futebol $(30 \%)$ e passou a caminhar $(10 \%)$, fazer alongamentos musculares (10\%), andar de bicicleta (10\%) e corrida (2\%) e notamos diminuição de praticantes do futebol (20\%). Com o grupo III notamos como ocorreu um grande interesse pela prática da atividade física, era um grupo de indivíduos que não se exercitavam, e com a intervenção de nosso programa, passou a dedicar um tempo do lazer à pratica do futebol $(50 \%)$, caminhada (6.6\%), alongamentos musculares $(10 \%)$, bicicleta $(10 \%)$, corrida (20\%) e natação (3.3\%).

FERRAREZI (1997) traçou o perfil 77 indivíduos, sendo 38 mulheres e 39 homens, frequentadores do parque da lagoa do Taquaral na cidade de Campinas, SP, .Utilizou um protocolo de avaliação com 22 questões abertas e fechadas. Os resultados mostraram que $50 \%$ realizam atividades físicas como forma de lazer, sendo em menos de 1 ano: $13.7 \%, 1$ a 5 anos: $43.8 \%$ e mais de 5 anos: $42.5 \%$. Tempo diário dedicado: até uma hora: $73.3 \%$ e mais de 1 hora: $26.7 \%$. Número de atividades: uma: $21.2 \%$, duas: $60.3 \%$ e três ou mais: $18.5 \%$.

Nos anos da década de 70, um projeto de grande envergadura tinha por objetivo estimular a prática da atividade física. O programa foi denominado de "Esporte para Todos", com apoio da televisão, por meio da chamada "Mexa-se". Esse colocou dezenas de pessoas nas ruas conscientizando-as da prática, até então, pouco difundida. Era o prenúncio da mudança de comportamento que assiste, no final da década de noventa, com parques lotados, as academias se proliferando e a consciência emergindo em um sentido de viver mais e melhor (SABA, 1998).

O projeto "Agita São Paulo", criado pelo governo estadual, tem conseguido atingir os seus objetivos, isto é, estimular as pessoas a gastar calorias com exercícios físicos. Semelhante ao programa de Atlanta, criado pelo Centro de Controle e Prevenção de Doenças, o programa oferece à população, atividades físicas, para estimular hábitos mais saudáveis. Idealizado pelo Centro de Estudos do Laboratório de Aptidão Física de São Caetano do Sul, o programa aborda vários grupos de pessoas, por meio de caminhada, palestras e jogos e consegue estimular as pessoas a realizarem atividades físicas, tanto sozinhas, como em grupos. Esse projeto, além de proporcionar melhora na qualidade de vida das pessoas participantes, proporciona outro elemento fundamental: a integração. Os indivíduos são motivados a desenvolver programas em conjunto com a sua comunidade e, é 
interessante observar, que tomam consciência de que a vida sedentária cria hábitos terríveis e danos irreparáveis à saúde (SABA, 1998).

\section{Conclução}

Atualmente o termo qualidade de vida vem sendo muito utilizado.

Vários estudos comprovam que a prática da atividade física, é um dos elementos fundamentais para a aquisição ou manutenção do estado da saúde, considerado adequado, tanto nos aspectos físico como mental. A atividade física, escolhida para ser praticada, deve ser aquela de que o indivíduo mais goste e que sinta prazer ao executá-la.

Apesar dos valores da prática da atividade física, uma grande parte da população brasileira, ainda é sedentária. Portanto, tornam-se necessárias campanhas e programas que divulguem os seus benefícios.
Os profissionais de Educação Física devem oferecer programas de atividades físicas que não proporcionem somente a vivência da prática da atividade, mas também, devem abordar aspectos teóricos que contribuam para o enriquecimento pessoal dos indivíduos.

Recomendamos a abordagem dos vários aspectos necessários à aquisição de qualidade de vida adequada. Sugerimos o enfoque da importância de termos hábitos saudáveis nas horas de lazer, ou seja, a prática da atividade física.

Concluímos que o programa foi eficiente, no sentido de conscientizar a população estudada, dos benefícios da prática da atividade física, tornando-os mais ativos fisicamente, tanto no trabalho, como principalmente no lazer. Observamos que os voluntários optaram pela prática, também, de outras atividades físicas, além do futebol, contribuindo para o aumento do repertório motor e melhoria da qualidade de vida da população estudada.

\section{Referências Bibliográficas}

ACHOUR,A. Jr..Efeitos das atividades físicas nos componentes herdados predisponentes a doenças cardiovasulares. Revista de Atividade Física e Saúde .v.1, n.4, p. 53-62,1996.

\section{AMERICAN HEART ASSOCIATION, SUBCOMMITTEE On EXERCISE/ CARDIAC REHABILITION. The} recommended quantitaty and quality of exercise for develeping nad maintaining fitness in healthy adults. Medicine Science Sports, v. 10, n. 3, 1981

AMORIM, P.R.S., et al. Distribuição de gordura corpórea como fator de risco no desenvolvimento de doenças arteriais coronarianas: Uma revisão de literatura. Revista de Atividade Física, v. 2 n.4, p. 59-79, 1997.

BLAIR, S. N. Research lecture: physical activity, fitness and hearth. Research Quaterly for Exercise and Sports, v.64, n.4, p.364-376, 1993.

BLAIR, S.N. Physical fitness and all cause mortality: a prospective study of healthy men and women. JAMA. V 262, p. 2395-2401, 1989.
BARBANTI, W. Aptidão Física e Saúde. Revista Festur, v. 3, n.1, p.5-8, 1991.

BARBOSA, J. A. et al. Um programa de atividade física para sedentários. In: Simpósio Internacional de Ciências do Esporte, 1998, São Paulo. Anais...São Paulo:115, 1998.

BAR-OR, O.; FOREYT, J; BOUCHARD, C. BROWNELL, K.D.; DIETZ, W.H; RAVUSSIN, E.; ARLINE, D; SALBE, A.D; SCHWENGER, S.; ST. JEOR, S. TORUN, $B$. Physical activity, genetic, and nutrition considerations in chidhood weight menagement. Medicine \& Sciencie in Sports \& Exercise, v. 30, n. 10, p. 2-10, 1998.

BERGER, B. MACINMAM, A. Exercise and the quality of life. In: SINGER, R., et al. Handbook of Researsh on Sport Physical. New York: Macmillan Publishing Company, 1993, cap. 34, p. 729-760.

BLUENTHAL, J.A.; EMERY,C.F.; WALSH, M.A.; COX, D.R. KUHN, C, M. WILLIAMS, R.B. Exercise training in healthy type A middle-aged men. Effects on behavioral and cardiovascular responses. Psychosomatic Medicine, 1988 , v. 50 , p. $418-433$. 
CAMPOS, I. S. L., POPOV, S. N. Exercícios físicos em terra e água. Uma proposta de prevenção e reabilitação. Belém : Supercores, 1998.

CARVALHO, T. Doenças crônicodegenerativas no Brasil In: Brasil, Ministério da Saúde -Coordenação de Doenças Crônico-Degenerativas. Orientações básicas sobre atividade física e saúde para os profissionais das áreas de educação saúde. Brasília: Ministério da Saúde, 1995.p.15-26.

CORBIN,C. \& LINDSEY, R. Concepts of physical fitness. Brown \& Bechmark Pubilshers, 1997.

CREWS, D.J. \& LANDERS, D.J. A metaanalitic review of aerobic fitness and reactivity of psychosocial stressors. Medicine Science and Exercise, v. 19 (suppl.), 1987, p. 114-120.

DISHMAN, R.K. Introduction: consensus, problems, and prospects. In DISHMAN, R.K., ed. Advances in exercise adherence. Champaign, Human Kinetics, 1994, Cap. 1, p.128.

Exercise adherence. In: SINGER, R.N. \& TENNANT, L.K., ed. Handbook of Research Sport Psychology. New York, McMillan, 1993, Cap. 36, p. 779-97.

DUMAZEDIER, J. Lazer e Cultura Popular. São Paulo : perspectiva, 1973.

FERRAREZI. A influência da atividade física na melhoria da qualidade de vida do homem. (Tese doutorado). Faculdade de Educação. UNICAMP, 1997.

FIATARONE,M.; MARKS,E.C.; RYAN, N.D.; MEREDITH,C.N. LIPSITZ, L.A.; EVANS, W.J. High intensity strenght training in nonagenarians. Journal of the American Medical Association., v. 263, p. 30293034, 1990.

GANDEE, R.N. et al. Stress, and older adults: a mind-body relationship. Journal of Physical Education, Recreation and Dance, v. 69, n. 9, p.19-22, 1988.

GUISELINI, M.A. Qualidade de vida: um programa prático para um corpo saudável. São Paulo : Gente, 1996.
GUTHRIE, JR, SMIHT, AMA, DENNERSTEIN, L. MORSE,C. Physical activity and menopause experience: a crosssectional study. Maatiritas, v. 20, p. 71, 1995.

KING, A.C.; TAYLOR, C.B.; HASKELL, W.L. Effects of differing intensities and formats of 12 months of exercise training on psychological outcomes in older adults. Helath Psychology, v. 12, n. 4, p. 292-300, 1993.

LITVAK, J., et al. The growing noncommunacable disease burden, a challenge for the conuntries of the Americas. Bull. Pan Am Health Organ, v. 21, p. 156-171, 1987.

MARCELLINO N.C. Lazer e educação. Campinas: Papirus, 1980.

MATSUDO, V. et al. Exercício e Qualidade de Vida. In: Congresso Latino Americano. Esporte, Educação e Saúde, Foz do Iguaçu, 1996. Anais... Foz do Iguaçu: 85-89, 1996.

MATSUDO, S.M.; MATSUDO, V.K.R.; ANDRADE, D.; ROCHA, J. Aptidão física e o gasto tempo assistindo TV em crianças de baixo nível sócio-cultural. Revista de Atividade Física, v.2, n. 1, p. 84, 1997.

MAZARINI, C. Natação para crianças portadoras de deficiência visual: Uma proposta de ensino. Campinas, 1992. Dissertação (mestrado)- Faculdade de Educação Física, 1992.

NAHAS, M.V. Esporte \& Qualidade de Vida. Revista da APEF, v.12, n. 2, 1997.

NIEMAN, D.C. The exercise-health connection. Champaign, Human Kinetics, 1998.

MEDEIROS, E.B. O lazer no planejamento urbano. Boletim de intercâmbio. Rio de Janeiro, v. 3, p. 37-54, 1980.

MOUSSA, M.A.A.; SHAIK, M.B.; SELWANES, S.B. YAGHY, O.Y.; BINOTHMAN, S.A. Factors associated with obesity in shoool children. International Journal of Obesity, v. 18, p. 513-15, 1994. 
NIH CONSENSUS CONFERENCE. Physical activity and cardiovascular health. JAMA, v. 276, p. 241-246, 1996.

RAMOS, M. G. Explorando as relações do grau de sedentarismo de alunos ingressantes na Universidade Estadual de Campinas. Campinas, 1998. Dissertação de Mestrado. Faculdade de Educação Física, UNICAMP, 1998.

RIGATTO, M. Preceitos para uma melhor qualidade de vida. Santa Maria: Departamento de Saúde Comunitária, 1994.

RIPPE, J.M. et al. Walking for healht and aptitude. AMA, Worcester, v. 13, n. 18, 1998.

REQUIXA, R. Sugestões de diretrizes para uma política nacional do lazer. São Paulo: Sesc, 1980.
SABA, F. A importância da atividade física para a sociedade e o surgimento das academias de ginástica. Revista de Atividade Física e Saúde, v. 2, n.2, p. 8087, 1998.

SAMULSKI, D. \& LUSTOSA, L. A importância da atividade física para a saúde e a qualidade de vida. Arthus- Revista de Educação Física e Desporto, v. 17, n. 1, p. 60-70, 1996.

SICHIERI, R. Epidemiologia da obesidade. Rio de Janeiro: Ed Uerj., 1998.

SINYOR， D. SCHWARTS, S.G.; PERONNET, F.; BRISSON, G. \& SERAGANIAN, P. Aerobic 1983).

STEPTOE, A. Aerobic exercise and health. In: KOELN; NITSCH,R. SEILER,R. (eds) Proceedings of the VIII Europena Congress of Sport Psychilogy 1991. Koeln Academia Verlag, v. 4, p. 78-91, 1994. 